\title{
Variabilidade horária do perfil de temperatura do solo em um pomar de mangueiras (Mangifera indica I.), na localidade de Cuiarana, Salinópolis - PA
}

\section{Romero Thiago Sobrinho Wanzeler ${ }^{1}$, José de Paulo Rocha da Costa ${ }^{2}$ e Cleber Assis dos Santos ${ }^{3}$}

1 Graduado em Meteorologia pela Universidade Federal do Pará (UFPA), Brasil. E-mail: romero-thiago@hotmail.com

2 Doutor em Meteorologia Agrícola pela Universidade Federal de Viçosa, Mestre em Meteorologia pela Universidade Federal da Paraíba, Especialista em Meteorologia Tropical na Universidade Federal do Pará e Graduado em Meteorologia pela Universidade Federal do Pará. Professor Associado III da Universidade Federal do Pará, Brasil. E-mail: jpaulo@ufpa.br

3 Especialista em Agriculturas Amazônicas e Desenvolvimento Agroambiental pelo Núcleo de Ciências Agrárias e Desenvolvimento Rural da UFPA, Graduado em Ciências Naturais com habilitação em Física pela Universidade do Estado do Pará. Cursando Bacharelado em Meteorologia pela Universidade Federal do Pará, Brasil. E-mail: cleberassis.ufpa@gamil.com

RESUMO: A temperatura do solo é um dos elementos mais importantes dentro da caracterização de um microclima. Este trabalho teve como objetivo analisar o perfil de temperatura no solo, em superfícies com solo vegetado e solo não vegetado, verificando a variação horária média da temperatura do solo em diferentes profundidades e comparando os perfis de temperatura do solo. Os dados horários de temperatura do solo foram medidos e coletados durante o período de 23 a 25 de junho de 2015, no sítio experimental Modesto Rodrigues, na localidade de Cuiarana, Salinópolis-PA. A temperatura do solo aumenta a partir das $6 \mathrm{~h}$ até aproximadamente às $14 \mathrm{~h}$, e diminui a partir desse horário. As maiores temperaturas ocorreram no solo não vegetado e as menores temperaturas no solo vegetado, devido a presença de maior umidade no solo vegetado. Pela análise dos resultados, concluiu-se que a temperatura do solo apresentou relação direta ao tempo de exposição à radiação solar, sendo este tipo de informação, imprescindível no entendimento de estudos agrometeorológicos e micrometeorológicos.

Palavras-chave: Amplitude térmica, radiação solar, variabilidade térmica.

Hourly variability of soil temperature profile in an orchard of mango trees (Mangifera indica I.) in the location of Cuiarana, Salinopolis - PA

ABSTRACT: The soil temperature is one of the most important elements in the characterization of a microclimate. The aim of this work was to analyze the temperature profile in the soil, on surfaces with vegetated and nonvegetated soil, checking the average hourly variation of soil temperature at different depths and comparing the soil temperature profiles. The hourly data of soil temperature were measured and collected during the period of 23 at 25 June of 2015, in the experimental site Modesto Rodrigues, in the locality of Cuiarana, Salinopolis-PA. The soil temperature increases from $6 \mathrm{~h}$ to approximately $14 \mathrm{~h}$, and decreases from that time. The highest temperatures occur in non-vegetated soil and the lower temperatures in the vege- 
tated soil, due to the presence of higher humidity in vegetated soil. In the analysis of results, the conclusion is that soil temperature was directly related to solar radiation exposure time, being this kind of information, essential in understanding agro-meteorological and micrometeorological studies.

Keywords: Thermal amplitude, solar radiation, thermal variability.

\section{INTRODUÇÃO}

Importantes processos e fenômenos físicos são objetos de estudo da micrometeorologia, principalmente os que ocorrem na camada da atmosfera mais baixa situada próximo à superfície da terra e que se estende verticalmente até alguns quilômetros. Uma importância peculiar é o conhecimento das variações com a altura da velocidade do vento, temperatura e concentração, em que por meio de modelos desenvolvidos sob condições idealizadas torna-se possível o estudo dos fluxos instantâneos de momentum, calor e massa (CAMPOS, 2004).

Varejão-Silva (2006) afirma que os estudos agrometeorológicos ou micrometeorológicos podem requerer a investigação do comportamento térmico do solo (para a determinação de fluxos de calor) em outras profundidades. Nos processos de interação solo-atmosfera os perfis de temperatura e umidade de solos dependem, dentre outros fatores, das características físicas e das trocas de calor e vapor d'água com a atmosfera que, por sua vez, dependem do clima e da cobertura vegetal local (ALVALÁ et al., 2002). A temperatura do solo é uma função da disponibilidade de radiação solar na superfície do solo e das suas propriedades térmicas, com efeito significativo sobre 0 crescimento e o desenvolvimento das plantas; suas funções metabólicas podem interagir na nutrição, absorção da água, na pro- dução dos compostos e no estoque de carboidratos (PARTON; LOGAN, 1981). Por outro lado, as propriedades térmicas do solo são resultantes de um conjunto de fatores nos quais se incluem sua textura e a composição química, e as observações regulares de sua temperatura em diferentes profundidades possibilitam identificar o seu comportamento térmico e o conhecimento de importantes propriedades, tais como difusividade e condutividade térmica (RAMANA RAO; SILVA; MOREIRA, 2005).

Segundo Castro (1989), a cobertura do solo reduz sua temperatura durante as horas mais quentes do dia. Existe uma faixa de temperatura favorável ao crescimento das plantas, podendo ser diferente para cada órgão ou fase de desenvolvimento desta. 0 aumento da temperatura acima deste ótimo pode prejudicar o metabolismo das plantas, ocorrendo em situações extremas a desnaturação de proteínas, inativação de enzimas e desidratação de células (SOUZA, 1996). Em solos cobertos as variações térmicas são pequenas, o teor de água é maior, enquanto que em solo nu ocorre maior amplitude de variação, podendo atingir altas temperaturas, inadequadas ao desenvolvimento radicular. A cobertura do solo evita seu aquecimento excessivo e é fator preponderante para a preservação e o acúmulo de matéria orgânica. Há, portanto, uma dupla ação da cobertura do solo: proteção e incorporação progressiva da matéria orgânica (MEDRADO; COSTA, 1990). 
Estudos de Shinzato (2009) mostraram que a influência da vegetação diminuiu a temperatura superficial em $23{ }^{\circ} \mathrm{C}$, medidos embaixo da copa das árvores, em relação a uma área descoberta com superfície em asfalto. Isso acontece pelo efeito do sombreamento das árvores com copa densa que evitam a chegada da radiação direta no solo, pelo efeito do dossel propriamente dito e pela cobertura do solo. Abaixo do dossel, as características do solo geram um conteúdo disponível de água para ocorrer o processo de evapotranspiração, reduzindo a temperatura das superfícies, principalmente no período do verão, assim como diminuir a quantidade de radiação de onda longa e o fluxo de calor sensível emitida para atmosfera (STONE, 2012). A temperatura do solo ainda influencia o crescimento do sistema radicular (NYE; TINKER, 1977), a absorção de íons e água (EPSTEIN, 1972) e a atividade microbiana (VOSS; SIDIRAS, 1985).

Diante dessas discussões, visando aumentar o conhecimento acerca da importância da temperatura do solo em vários processos fisiológicos, o objetivo deste trabalho foi analisar o perfil de temperatura no solo, em condições de cobertura do solo vegetado e não vegetado, verificando a variação horária da temperatura do solo em diferentes profundidades e comparando esses perfis de temperatura do solo nas duas condições de solo definidas anteriormente.

\section{MATERIAIS E MÉTODOS}

Os dados utilizados nesta pesquisa foram coletados durante de experimento micrometeorológico, realizado no sítio experimental Modesto Rodrigues, na localidade de Cuiarana, em Salinópolis - PA (Lat. $00^{\circ}$ $39^{\prime} 45^{\prime \prime}$ S, Long. $47^{\circ} 16^{\prime} 56^{\prime \prime} \mathrm{O}$ ), identificada na Figura 1. As medições e a coleta dos dados ocorreram no período de 23 a 25 de junho de 2015. O pomar de mangueiras (Mangifera Indica L.) foi plantado para fins comerciais e para exportação, a cerca de 10 anos, em fileiras orientadas no sentido Leste-Oeste, com espaçamento de aproximadamente 10 metros entre fileiras (SOUZA JúNIOR et al., 2010).

Figura 1 - Mapa da localização geográfica de Cuiarana, Salinópolis - PA.

Figure 1 - Map of the geographical location of Cuiarana, Salinópolis - PA.

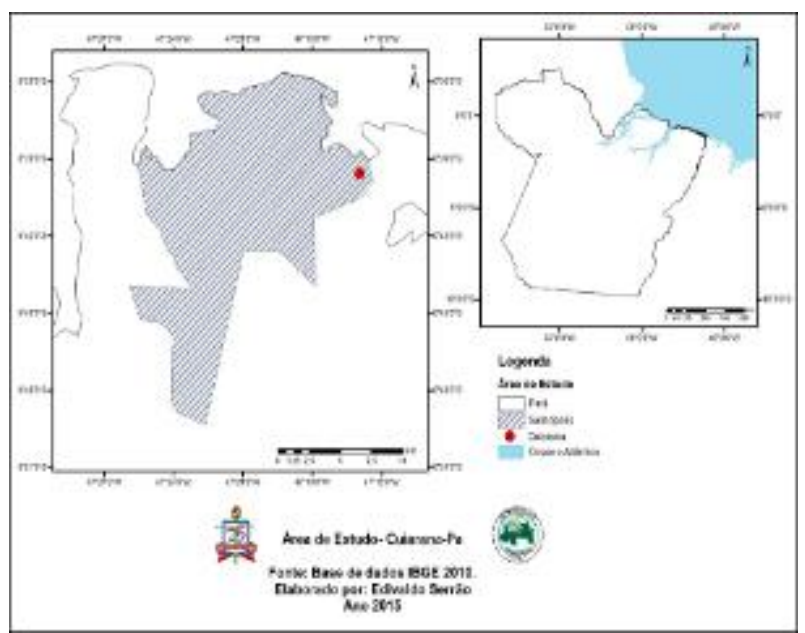

Fonte: Do Autor.

Para registrar os valores de temperatura do solo, foram utilizadas duas baterias de geotermômetros, uma em solo com vegetação (Figura 2.a) e outra em solo sem vegetação (Figura 2.b) , instalados nos seguintes níveis: $10 \mathrm{~cm}$ acima da superfície, termômetro de relva na superfície do solo (Figura 2.c), $2 \mathrm{~cm}, 5 \mathrm{~cm}$ e $10 \mathrm{~cm}$ abaixo da superfície. Também foram utilizados Termistores (sensores de temperatura do solo), idênticos ao da Figura 2.d, para medições automáticas da temperatura do solo a $2 \mathrm{~cm}, 20$ $\mathrm{cm}$ e $50 \mathrm{~cm}$ abaixo da superfície do solo, 
conectados a um Datalloger CR10X, da Campbell Scientific (Figura 2.e).

Figuras 2 - 2.a. Bateria de geotermômetros em solo com vegetação; Figura 2.b. Bateria de geotermômetros em solo sem vegetação; Figura 2.c. Termômetro de relva na superfície do solo; Figura 2.d. Sensor de temperatura do solo (Termistor); Figura 2.e. Datalloger CR10X, da Campbell Scientific.

Figure 2 - 2.a. Battery of geothermometers in soil with vegetation; Figure 2.b. Battery of geothermometers in bare soil; Figure 2.c. grass thermometer on the soil surface; Figure 2.d soil temperature sensor (thermistor); Figure 2.e. Datalloger CR10X, Campbell Scientific.

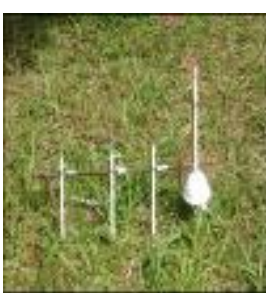

(2.a)

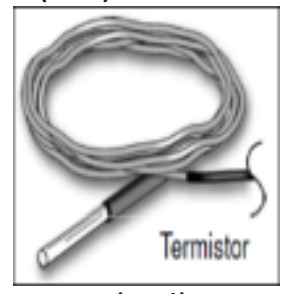

(2.d)

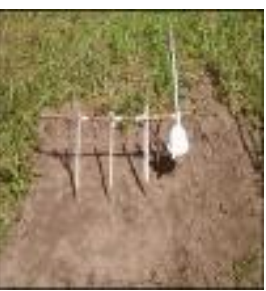

(2.b)

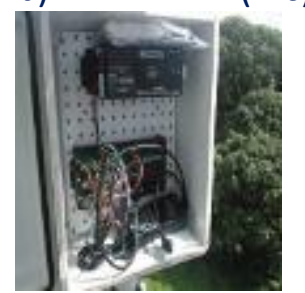

(2.e)

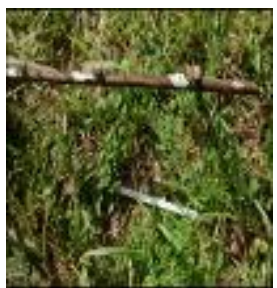

(2.c)
Fonte: Do Autor.

Após a coleta, realizada de hora em hora, esses dados eram repassados à planilha eletrônica do Excel e verificados quanto à sua consistência, para a elaboração de gráficos, apresentando a variação horária média da temperatura do solo em diferentes profundidades sob duas diferentes condições de solo, durante o período do trabaIho.

\section{RESULTADOS E DISCUSSÃO}

As variações médias da temperatura do solo ao longo do período de 23 a 25 de junho de 2015 e das profundidades, no solo com vegetação e no solo sem vegetação, são apresentadas nas tautócronas abaixo.
No solo com vegetação (Figura 3 ), a temperatura atingiu valores máximos em todas as profundidades entre às $14 \mathrm{~h}$ e $15 \mathrm{~h}$, sendo que a maior amplitude térmica ocorreu no nível de superfície e a menor foi verificada na profundidade de $50 \mathrm{~cm}$ abaixo do solo, relacionada a grande concentração de umidade no solo neste nível e a amenização do regime térmico no solo causada pela vegetação.

Figura 3 - Tautócrona de temperatura média do solo com vegetação (geotermômetros + termistores), durante o período de 23 a 25 de junho de 2015.

Figure 3 - Tautocrona of average soil temperature with vegetation (geothermometers + thermistors), during the period from 23 to 25 June 2015.

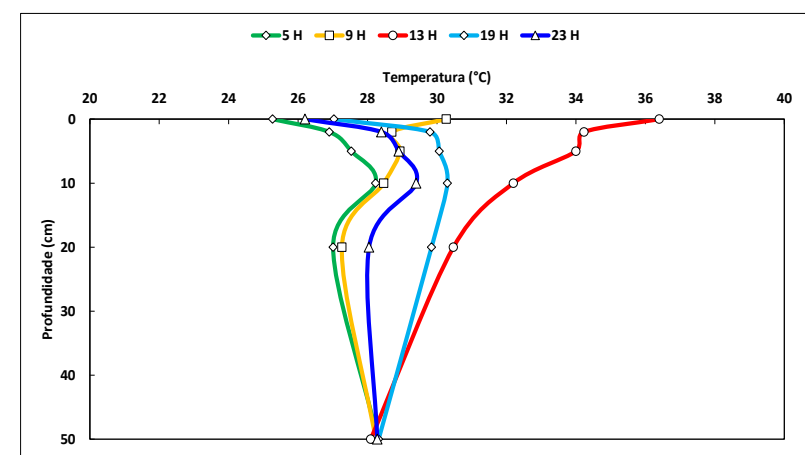

Fonte: Do Autor.

No solo sem vegetação (Figura 4), em todas as profundidades a temperatura máxima ocorreu no horário das $14 \mathrm{~h}$, e as amplitudes térmicas foram maiores do que no solo com vegetação, principalmente a $2 \mathrm{~cm}$ abaixo do solo, uma vez que a ausência de cobertura vegetal altera o balanço de energia e a radiação solar atinge diretamente 0 solo. Pezzopane et al. (1996), encontrou resultados semelhantes em estudo em solo nu e em solo coberto com palha de café (material isolante térmico), e mostrou que houve maior amplitude térmica no solo nu, e com a temperatura nas horas mais quentes atingindo mais de $45{ }^{\circ} \mathrm{C}$ a $2 \mathrm{~cm}$ de profundidade. $\mathrm{O}$ mesmo autor verificou que, à 
medida que a quantidade de palha de café foi aumentando, a amplitude diária foi diminuindo.

Figura 4 - Tautócrona de temperatura média do solo, sem vegetação, durante o período de 23 a 25 de junho de 2015.

Figure 4 - Tautocrona of average soil temperature without vegetation during the period from 23 to 25 June 2015 .

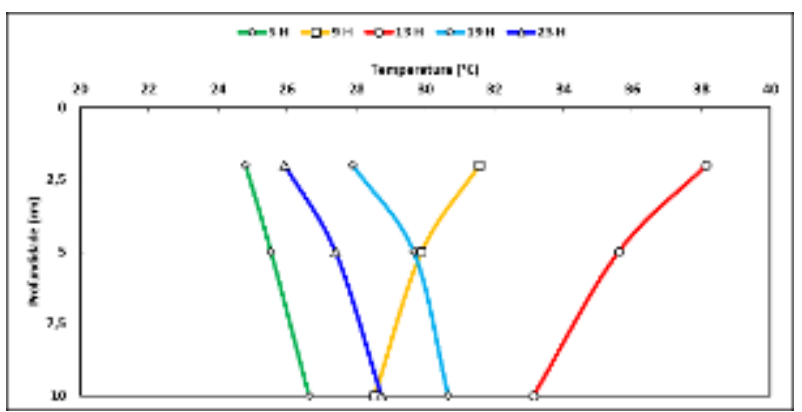

Fonte: Do Autor.

A Figura 5 mostra a variação média da temperatura a $10 \mathrm{~cm}$ acima do solo. No ar que se encontra próximo ao solo, os processos responsáveis pelas trocas de calor são a condução e a convecção de calor. Este último é o principal responsável pelo aquecimento do ar próximo ao solo (BÍSCARO, 2007). Ocorre um aumento da temperatura do ar que se encontra próximo ao solo, a partir das $6 \mathrm{~h}$ e pela manhã, até alcançar o seu valor máximo às $12 \mathrm{~h}$ no solo com vegetação e às $13 \mathrm{~h}$ no solo sem vegetação. A partir destes horários o ar passa a perder calor, e consequentemente, ocorre a diminuição da temperatura.
Figura 5 - Variação média da temperatura à $10 \mathrm{~cm}$ próximo do solo, durante o período de 23 a 25 de junho de 2015 .

Figure 5 - Average change of temperature at $10 \mathrm{~cm}$ close to the soil, during the period from 23 to 25 June 2015.

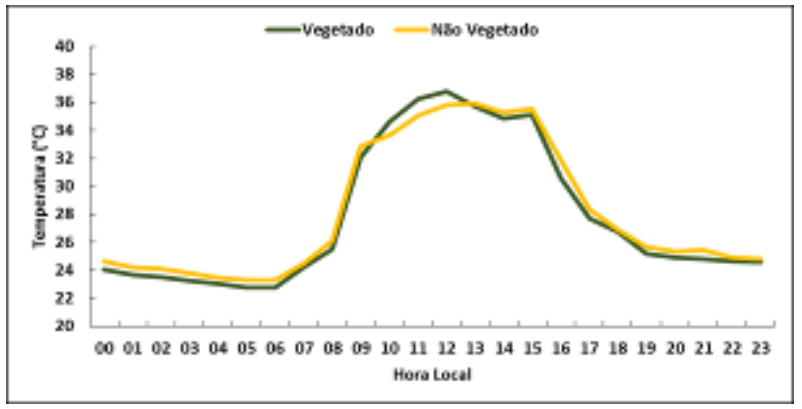

Fonte: Do Autor.

Verificou-se que nos horários de $10 \mathrm{~h}$ às $12 \mathrm{~h}$, ocorreu uma inversão nas curvas de temperatura do solo, passando a temperatura no solo vegetado a ser mais quente do que no solo não vegetado, e uma possível explicação para esta alteração no padrão, pode ser o fato de que durante esses horários ocorria um sombreamento no termômetro em solo não vegetado, causado por uma árvore que se encontrava nas proximidades do local onde os termômetros encontravam-se instalados.

Na Figura 6 é apresentada a variação média da temperatura do solo no nível de 2 $\mathrm{cm}$ abaixo do solo, em superfícies vegetada e não vegetada, e no nível de superfície, com termômetro de relva. A temperatura máxima foi verificada nessas três situações às $14 \mathrm{~h}$, e a maior amplitude térmica ocorreu no solo sem vegetação, onde a radiação de onda curta incide diretamente no solo, e a temperatura atinge valores mais altos. Nye e Tinker (1977) afirmam que em solo não vegetado, devido à maior amplitude de variação, as altas temperaturas podem se tornar inadequadas ao desenvolvimento radicular. Durante a madrugada e a noite, verificou-se uma proximidade entre os valores de temperatura da relva e de $2 \mathrm{~cm}$ abai- 
xo do solo não vegetado, e em ambos, a temperatura mínima ocorreu às $6 \mathrm{~h}$.

Figura 6 - Variação média da temperatura à $2 \mathrm{~cm}$ abaixo do solo, em superfície vegetada e não vegetada e sobre a relva durante o período de 23 a 25 de junho de 2015.

Figure 6 - Average change in temperature at $2 \mathrm{~cm}$ below the soil, in vegetated surface and non-vegetated and on the grass during the period from 23 to 25 June 2015.

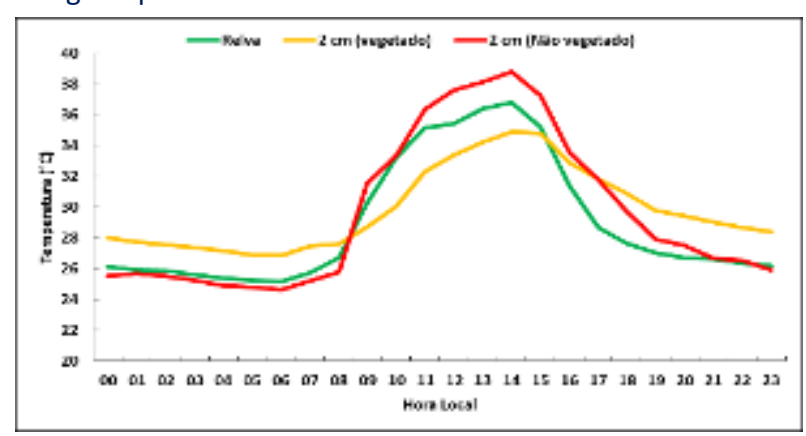

Fonte: Do Autor.

As variações médias da temperatura do solo nos níveis de $2 \mathrm{~cm}, 5 \mathrm{~cm}$ e $10 \mathrm{~cm}$ abaixo do solo vegetado e não vegetado, são mostradas na Figuras 7.a, 7.b e 7.c, respectivamente. Percebe-se o regime térmico característico de um solo, determinado pelo aquecimento que ocorre na superfície pela radiação solar, e pelo transporte de calor sensível ao seu interior, através do processo de condução. Ocorre durante o dia, o aquecimento da superfície, originando um fluxo de calor da superfície para o interior do solo, aumentando o armazenamento de energia e consequentemente, elevando a sua temperatura. À noite, devido o resfriamento da superfície, por emissão de radiação terrestre, há diminuição da temperatura nas camadas próximas à superfície, e isto inverte o sentido do fluxo de calor, que se torna ascendente, retornando o calor armazenado para a superfície.
Figura 7 - 7.a. Variação média da temperatura do solo à $2 \mathrm{~cm}$ abaixo da superfície; Figura 7.b. Variação média da temperatura do solo à $5 \mathrm{~cm}$ abaixo da superfície; Figura 7.c. Variação média da temperatura do solo à $10 \mathrm{~cm}$ abaixo da superfície.

Figure $7-7 . a$. Average change of soil temperature at $2 \mathrm{~cm}$ below the surface; Figure 7.b. Average change of soil temperature at $5 \mathrm{~cm}$ below the surface; Figure 7.c. Average change of soil temperature at $10 \mathrm{~cm}$ below the surface.

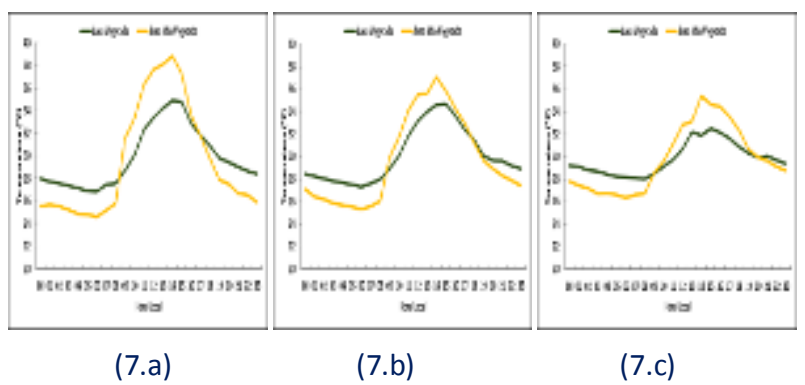

Fonte: Do Autor.

As Figuras 8.a e 8.b abaixo apresentam o ciclo diário da temperatura do solo à $2 \mathrm{~cm}$, $5 \mathrm{~cm}$ e $10 \mathrm{~cm}$ abaixo do solo, em superfícies vegetada e não vegetada, respectivamente, que ocorreu no período de 23 a 25 de junho de 2015. Nas duas superfícies, é possível verificar que a temperatura do solo varia de acordo com o balanço de radiação, que é positivo durante o dia, uma vez que o aquecimento da superfície origina um fluxo que transporta calor da superfície para o interior do solo, aumentando o armazenamento de energia com consequente elevação da sua temperatura, que também aumenta de acordo com a posição do sol, atingindo valores máximos sempre às $14 \mathrm{~h}$. Durante a noite e pela madrugada a temperatura diminui, atingindo valor mínimo às $6 \mathrm{~h}$, devido o resfriamento da superfície, por emissão de radiação terrestre, que inverte o sentido do fluxo de calor, que se torna ascendente, retornando o calor armazenado para a superfície. 
Figura 8 - 8.a. Ciclo diário da variação da temperatura do solo nos níveis de $2 \mathrm{~cm}, 5 \mathrm{~cm}$ e $10 \mathrm{~cm}$, em solo vegetado, no período de 23 a 25 de junho de 2015; Figura 8.b. Ciclo diário da variação da temperatura do solo nos níveis de $2 \mathrm{~cm}, 5 \mathrm{~cm}$ e $10 \mathrm{~cm}$, em solo não vegetado, no período de 23 a 25 de junho de 2015 .

Figure $8-8$.a. Daily cycle of soil temperature variation in the levels of $2 \mathrm{~cm}, 5 \mathrm{~cm}$ and $10 \mathrm{~cm}$, in vegetated soil, in the period of 23 to 25 June 2015.; Figure 8.b. Daily cycle of soil temperature variation in the levels of $2 \mathrm{~cm}, 5 \mathrm{~cm}$ and $10 \mathrm{~cm}$, in nonvegetated soil, in the period of 23 to 25 June 2015.

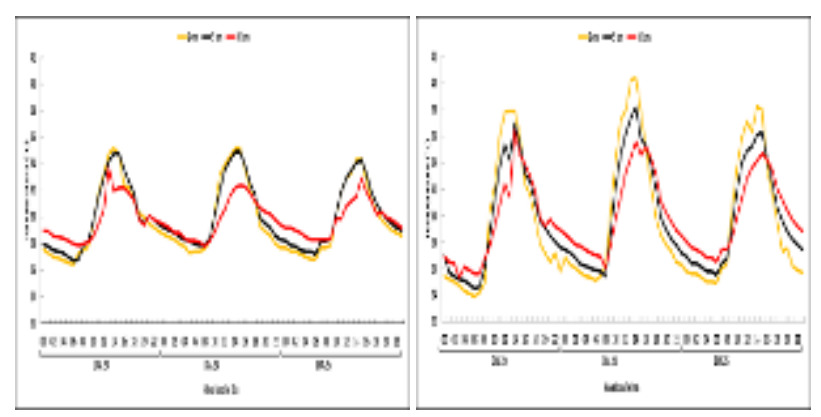

(8.a)

(8.b)

Fonte: Do Autor

Os maiores valores de amplitude térmica foram encontrados no solo não vegetado, sendo que no dia 24 , ocorreu a maior variação durante o período de estudos, que foi de $15,3{ }^{\circ} \mathrm{C}$. Em todos os níveis do solo não vegetado, a amplitude térmica foi superior ao solo vegetado e os menores valores de amplitude $\left(4,6{ }^{\circ} \mathrm{C}\right)$ foram verificadas no nível de $10 \mathrm{~cm}$ abaixo do solo com vegetação, nos dias 24 e 25 . Isso está relacionado ao conteúdo de umidade presente no solo da área de estudos, semelhante a resultados encontrados por Costa et al (2002) em estudos realizados na floresta de CaxiuanãPA.

\section{CONCLUSÃO}

A análise e a discussão dos resultados acima, evidenciaram que nos dias de realização da pesquisa deste trabalho, na localidade de Cuiarana, em Salinópolis - PA, a temperatura do solo esteve mais alta durante os horários de maior luminosidade, logo ela esteve relacionada diretamente ao tempo de exposição à radiação solar, com ocorrência de maiores temperaturas no solo não vegetado e menores temperaturas no solo vegetado. $\mathrm{O}$ solo vegetado apresentou menores amplitudes térmicas de variação em relação ao solo não vegetado, o que esteve relacionado à interceptação da radiação solar antes de atingir o solo. A amplitude térmica foi maior nas menores profundidades e menor nas maiores profundidades, por influência direta da incidência da radiação solar.

\section{REFERÊNCIAS}

ALVALÁ, R. C. S. et al. Intradiurnal and seasonal variability of soil temperature, heat flux, soil moisture content and thermal properties under forest and pasture in Rondonia. Journal of Geophysical Research - Atmospheres, v. 107, p. 10-20, 2002. BÍSCARO, G. A. Meteorologia Agrícola Básica. UNIGRAF. Mato Grosso do Sul. 83 p. 2007.

CAMPOS, C. R. J. Micrometeorologia: Tópicos Gerais. Editora e gráfica UniversitáriaUFPEL, 2004.

CASTRO, O. M. Preparo do solo para a cultura do milho. Campinas, Fundação Cargill, 1989.

COSTA, J. P. R. et al. Variação média do curso diário da insolação e temperatura do ar e do solo em área de clareira na floresta Amazônica. In: CONGRESSO BRASILEIRO DE METEOROLOGIA, 12, 2002. Anais. Foz do Iguaçu: SBMET, 2002.

EPSTEIN, E. Mineral nutrition of plants: principles and perspectives. New York. John Willey Ed. 1972.

MEDRADO, M. J. S.; COSTA, J. D. Clones de seringueira para a região do planalto pau- 
lista. Piracicaba, PCAP/USP. Informativo técnico no 9, 12 p. 1990.

NYE, P. H.; TINKER, P. B. Solute movement in the soilroot system. Oxford, Blackwel. 1977.

PARTON, W. J.; LOGAN, J. A. A model for diurnal variation in soil and in temperature. Agricultural Meteorology, v.23, p. 205-216, 1981.

PEZZOPANE, J. E. M. et al. Temperatura do solo em função da cobertura morta por paIha de café. Revista Brasileira de Agrometeorologia, v. 4 (2), p. 7-10, 1996.

RAMANA RAO, T. V.; SILVA, B. B.; MOREIRA, A. A. Características térmicas do solo em Salvador, BA. Revista Brasileira de Engenharia Agrícola e Ambiental, v. 9, p. 554559, 2005.

SHINZATO, P. Impacto da vegetação nos microclimas urbanos. Dissertação (Mestrado em Arquitetura). Faculdade de Arquitetura e Urbanismo, Universidade de São Paulo, 2009.

SOUZA JÚNIOR, J. A. et al. Microclima em um pomar de mangueiras: Cuiarana - Salinas - Pará. In: CONGRESSO BRASILEIRO DE METEOROLOGIA, 16, 2010. Anais. Belém: SBMET, 2010.

SOUZA, M. M. S. R. Ação da cobertura com leguminosas sobre o solo e o seringal em formação. Dissertação (Mestrado). Escola Superior de Agricultura "Luiz de Queiroz", Universidade de São Paulo, 1996.

STONE, B. The City and the Coming Climate: Climate Change in the Places We Live. New York: Cambridge University Press, 2012.

VAREJÃO-SILVA, M. A. Meteorologia e Climatologia. Versão Digital 2. Recife, Pernambuco. Brasil. 2006. Disponível em: $<$ http://www.agritempo.gov.br/publish/pu
blicacoes/livros/METEOROLOGIA_E_CLI MATOLOGIA_VD2_Mar_2006.pdf>. VOSS, M.; SIDIRAS, N. Nodulação da soja em plantio direto em comparação com plantio convencional. Pesquisa Agropecuária Brasileira, v. 20, p. 775-782, 1985.

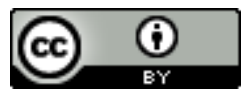
License information: This is an openaccess article distributed under the terms of the Creative Commons Attribution License, which permits unrestricted use, distribution, and reproduction in any medium, provided the original work is properly cited.

Artigo recebido em 17 de agosto de 2015.

Avaliado em 18 de fevereiro de 2016.

Aceito em 10 de março de 2016.

Publicado em 25 de maio de 2016.

Como citar este artigo (ABNT):

WANZELER, Romero Thiago Sobrinho; COSTA, José de Paulo Rocha da; SANTOS, Cleber Assis dos. Variabilidade horária do perfil de temperatura do solo em um pomar de mangueiras (Mangifera indica I.), na localidade de Cuiarana, Salinópolis-PA. Estação Científica (UNIFAP), Macapá, v. 6, n. 1, p. 117-124, jan./abr. 2016. 